\title{
Revalidation and redescription of Bungona illiesi (Lugo-Ortiz \& McCafferty) (Ephemeroptera: Baetidae) from Australia, based on mitochondrial and morphological evidence
}

\author{
J.M. WEBB ${ }^{1,3} \&$ P.J. SUTER ${ }^{2,3}$ \\ ${ }^{1}$ Department of Environmental Management and Ecology, La Trobe University, P.O. Box 821, Wodonga, Victoria, Australia 3689. \\ E-mail: jmw975@yahoo.com \\ ${ }^{2}$ Department of Environmental Management and Ecology, La Trobe University, P.O. Box 821, Wodonga, Victoria, Australia 3689. \\ E-mail:p.suter@latrobe.edu.au \\ ${ }^{3}$ Taxonomy Research \& Information Network
}

\begin{abstract}
Bungona illiesi (Lugo-Ortiz \& McCafferty) n.comb. was described from a single specimen collected in northern Queensland, Australia, as a species of Cloeodes Traver. The distinguishing characteristics of a minute maxillary palp, the absence of spination on the mandibles between the mola and prostheca, narrow-elongate gills, and medially expanded labial palps were later found to occur in Bungona narilla Harker, and the two species were synonymized. Extensive collecting in northern Queensland yielded larvae and adults of Bungona that differ from B. narilla in all life stages. We believe our specimens are equivalent to $B$. illiesi because they were collected only a few kilometres from the type locality, at least one specimen had both minute and normally sized maxillary palps, nearly all specimens had medially expanded labial palps, and the dorsal abdominal colour pattern was similar. Sequences of a 657-bp fragment of the mitochondrial gene cytochrome oxidase I supported the recognition of two species and confirmed the association of larvae and adults of $B$. illiesi. Interspecific p-distances ranged from 18.8-19.4\% and the maximum intraspecific divergence observed was $1.7 \%$. Based on the combination of morphological, mitochondrial, and biogeographical data, we show $B$. illiesi should be recognised as a valid species distinct from $B$. narilla. Larvae of $B$. illiesi differ from those of $B$. narilla by having fewer robust setae on the femora and a small lateral tooth on the outer incisor of both mandibles. Male and female adults differ from those of $B$. narilla by having less extensive red colouration on the abdominal terga.
\end{abstract}

Key words: mayfly, Australia, mtDNA, cytochrome oxidase I, taxonomy

\section{Introduction}

The Australian Baetidae remain poorly known, with only 20 species described to date, although unpublished data indicate the occurrence of approximately 60 species. Bungona Harker was established for B. narilla Harker, 1957 and described from larvae, males, and female subimagos from near Sydney, New South Wales. Adults of Bungona are distinguished from those of other Australian baetids by the absence of hindwings, turbinate eyes of the male with parallel margins, double marginal intercalaries in the forewing, a distinctive colour pattern, and the lack of a dorsal projection on the metascutellum. The larvae are differentiated from those of all other Australian Baetidae by the presence of transverse rows of fine setae on abdominal sterna 46 , an arc of very long, fine setae on the tibiae, and an oblique row of very long, fine setae on the tarsi.

Since its initial description, Bungona has been found to be widespread in eastern Australia from northern Queensland to Victoria, and one specimen is known from Tasmania (Suter and Pearson 2001). In their redescription of Bungona, Suter and Pearson (2001) synonymized two species that had been described in Cloeodes Traver by Lugo-Ortiz and McCafferty (1998) with B. narilla. Cloeodes fustipalpus Lugo-Ortiz \& McCafferty, 1998 was described from a small series of larvae and exuviae from northern New South Wales, and C. illiesi Lugo-Ortiz \& McCafferty, 1998 was based on a single specimen from near Cairns in northern Queensland. 
Suter and Pearson (2001) clearly showed that C. fustipalpus and B. narilla were conspecific based on morphological characters and biogeography. Because $C$. illiesi was described from a single specimen, it is difficult to ascertain whether it represents an aberrant specimen of $B$. narilla or is a distinct species. The main diagnostic characteristics for $C$. illiesi identified by Lugo-Ortiz and McCafferty (1998) include a minute second segment of the maxillary palp, the absence of minute spines between the mola and prostheca on the mandibles, bulbous labial palp segment 3, and narrow-elongate gills. As all of these characteristics were found to also occur in some specimens of B. narilla, Suter and Pearson (2001) placed C. illiesi as a junior synonym of $B$. narilla. The type locality of $C$. illiesi, however, is in northern Queensland north of the Burdekin Gap, a well known biogeographic division in aquatic insects (i.e. Watson and Theischinger 1984; Christidis and Dean 2008), and C. illiesi may be a distinct species from B. narilla.

In this paper, we show that all Bungona from northern Queensland have fixed morphological and mitochondrial character states and extensive mitochondrial sequence divergence from those occurring elsewhere in Australia. Based on morphological characters and proximity to the type locality, we conclude that the northern specimens are equivalent to C. illiesi and that it should be placed in Bungona. The larvae are redescribed and the adult stages described for the first time.

\section{Methods}

Larvae and adults of Bungona were collected from throughout its known range except Tasmania. All recently collected material was fixed and preserved in $95-100 \%$ ethanol, and some specimens were temporarily stored in 100\% propylene glycol. Larvae were collected with kick nets, and adults were collected using black lights. Slides preparations were made using Euparal; prior to being placed in Euparal, parts were soaked briefly in cellosolve (ethylene glycol monoethyl ether) to remove any water.

Twelve specimens were used for molecular analyses; eight specimens were from two localities in northern Queensland and the remaining four specimens were from southeast Queensland, northern New South Wales and southern New South Wales. Total genomic DNA was extracted using a proteinase-K/Chelex solution. A whole specimen or a small amount of thoracic muscle was placed in $100 \mu 1$ of Chelex solution (containing $5 \%$ Chelex (weight/volume) , $0.2 \%$ SDS, $10 \mathrm{mM}$ Tris $\mathrm{pH} 8,0.5 \mathrm{mM}$ EDTA) and $10 \mu \mathrm{l}$ of $20 \mathrm{mg} / \mathrm{mL}$ proteinase-K, incubated overnight at $55^{\circ} \mathrm{C}$, centrifuged for 5 minutes at $1500 \mathrm{rpm}$ and incubated for $5 \mathrm{~min}$ at $95^{\circ} \mathrm{C}$ to deactivate the proteinase-K. Prior to centrifuging, exuviae were removed and returned to alcohol or mounted on a microscope slide with Euparal and cellosolve. A portion of the DNA extraction was diluted 1 in 5 with $1 \mathrm{X}$ TE and used for subsequent analyses to reduce the number of freeze/thaw cycles on the original extraction. Undiluted extractions were stored at $-80^{\circ} \mathrm{C}$ and diluted extractions were stored at $-20^{\circ} \mathrm{C}$.

A 658-bp fragment of the mitochondrial gene cytochrome oxidase I (COI) was amplified using the primers LCO149 and HCO2198 (Folmer et al., 1994); primers were M13-tailed to facilitate sequencing. PCR for COI reactions consisted of $3.5 \mu \mathrm{l}$ buffer, $17.5 \mu \mathrm{l} 10 \% \mathrm{w} / \mathrm{v}$ trehalose, $0.7 \mu \mathrm{dNTPs}, 1.75 \mu \mathrm{l} 50 \mathrm{mM} \mathrm{MgCl}_{2}$, $0.7 \mu \mathrm{l}$ of each primer, $0.125 \mu \mathrm{l}$ taq polymerase (Invitrogen), $0.01-5 \mu \mathrm{l}$ of DNA template, and water to $35 \mu \mathrm{l}$. PCR conditions consisted of $1 \mathrm{~min}$ at $94{ }^{\circ} \mathrm{C} ; 5$ cycles of $1 \mathrm{~min}$ at $94{ }^{\circ} \mathrm{C}, 1.5 \mathrm{~min}$ at $45^{\circ} \mathrm{C}, 1.5 \mathrm{~min}$ at $72{ }^{\circ} \mathrm{C} ; 35$ cycles of $1 \mathrm{~min}$ at $94{ }^{\circ} \mathrm{C}, 1 \mathrm{~min}$ at $50^{\circ} \mathrm{C}, 1 \mathrm{~min}$ at $72{ }^{\circ} \mathrm{C}$; and 4 min at $72{ }^{\circ} \mathrm{C}$.

PCR products were purified and sequenced in both directions by Macrogen Inc (Seoul, Korea). Contigs were assembled in DNABaser 2.75 (www.DnaBaser.com) and aligned with default settings in Clustal X as implemented in MEGA4 (Tamura et al. 2007). All sequences were examined for the presence of double peaks, frame shifts and stop codons. Prior to analysis the first base was removed so all sequences started on the first codon position. Sequences were submitted to GenBank under accession numbers HM017831HM017842. Pairwise p-distances were calculated in MEGA4 and used to calculate mean, minimum, and maximum inter- and intraspecific divergence. A Neighbour-Joining (NJ) analysis was performed in MEGA4 using Kimura 2 Parameter distance. Maximum Parsimony (MP) analyses were performed in PAUP* 4.0b10 (Swofford 2002) with 10 random sequence addition replicates and tree-bisection-reconnection (TBR) branch 


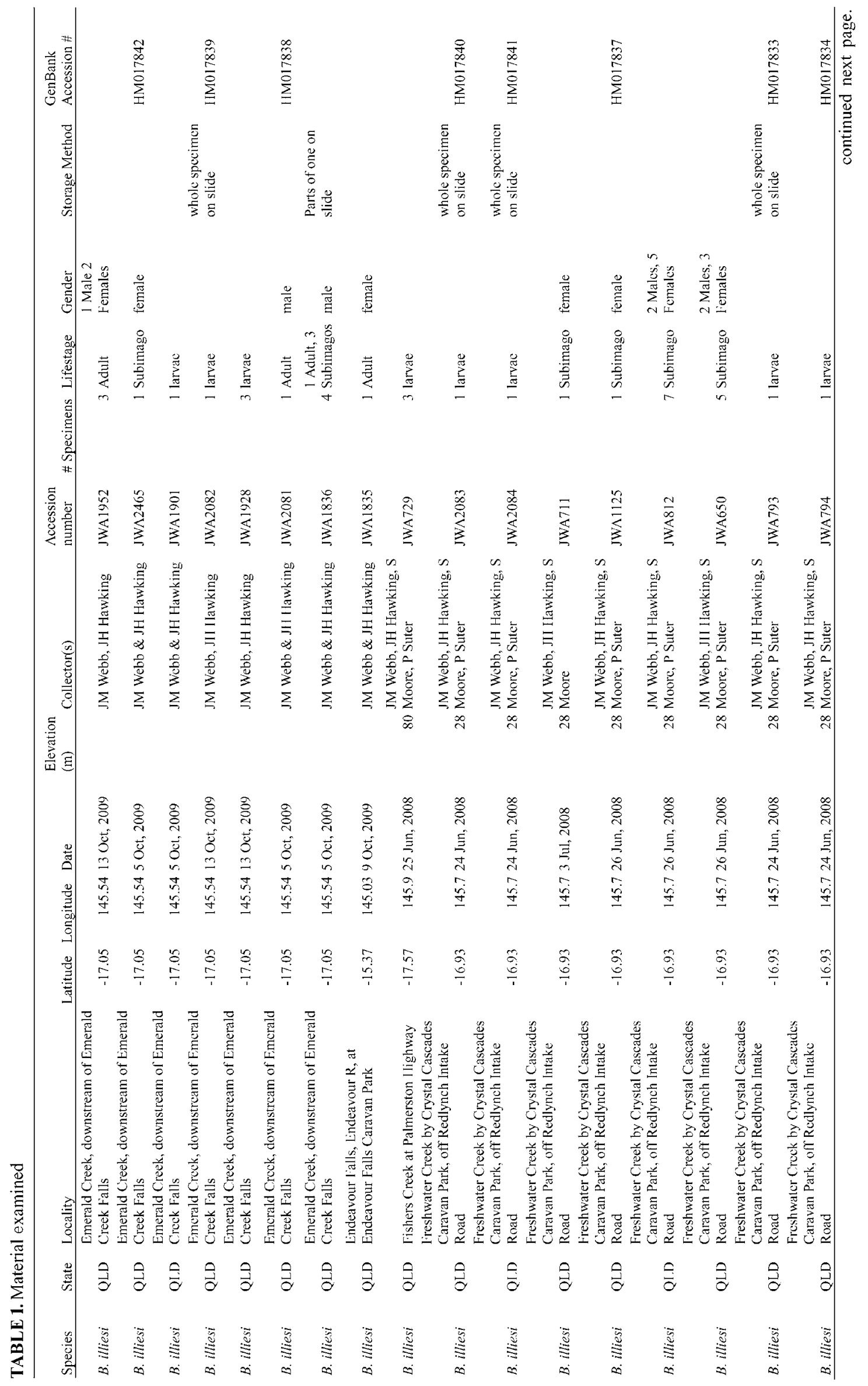




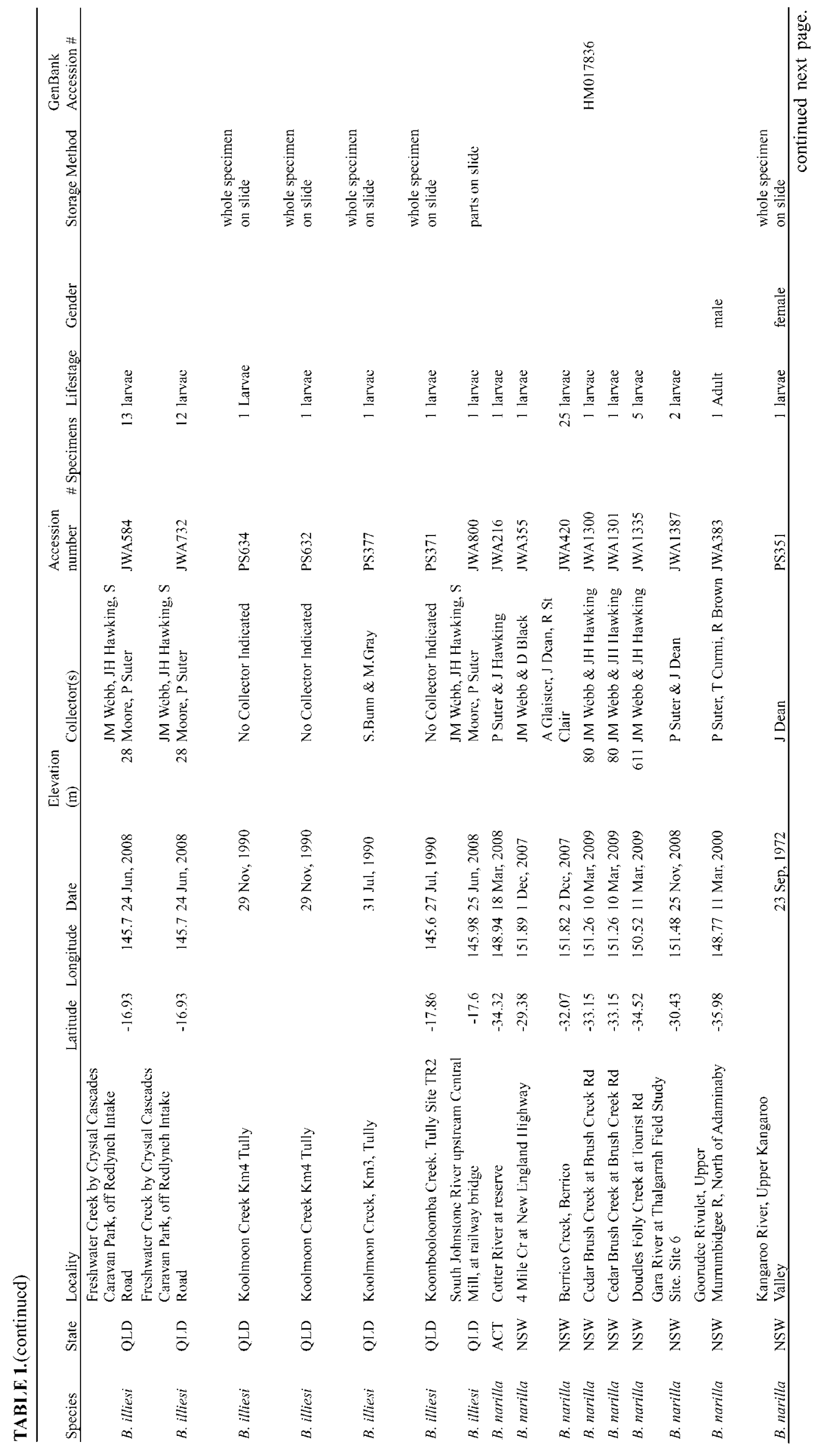




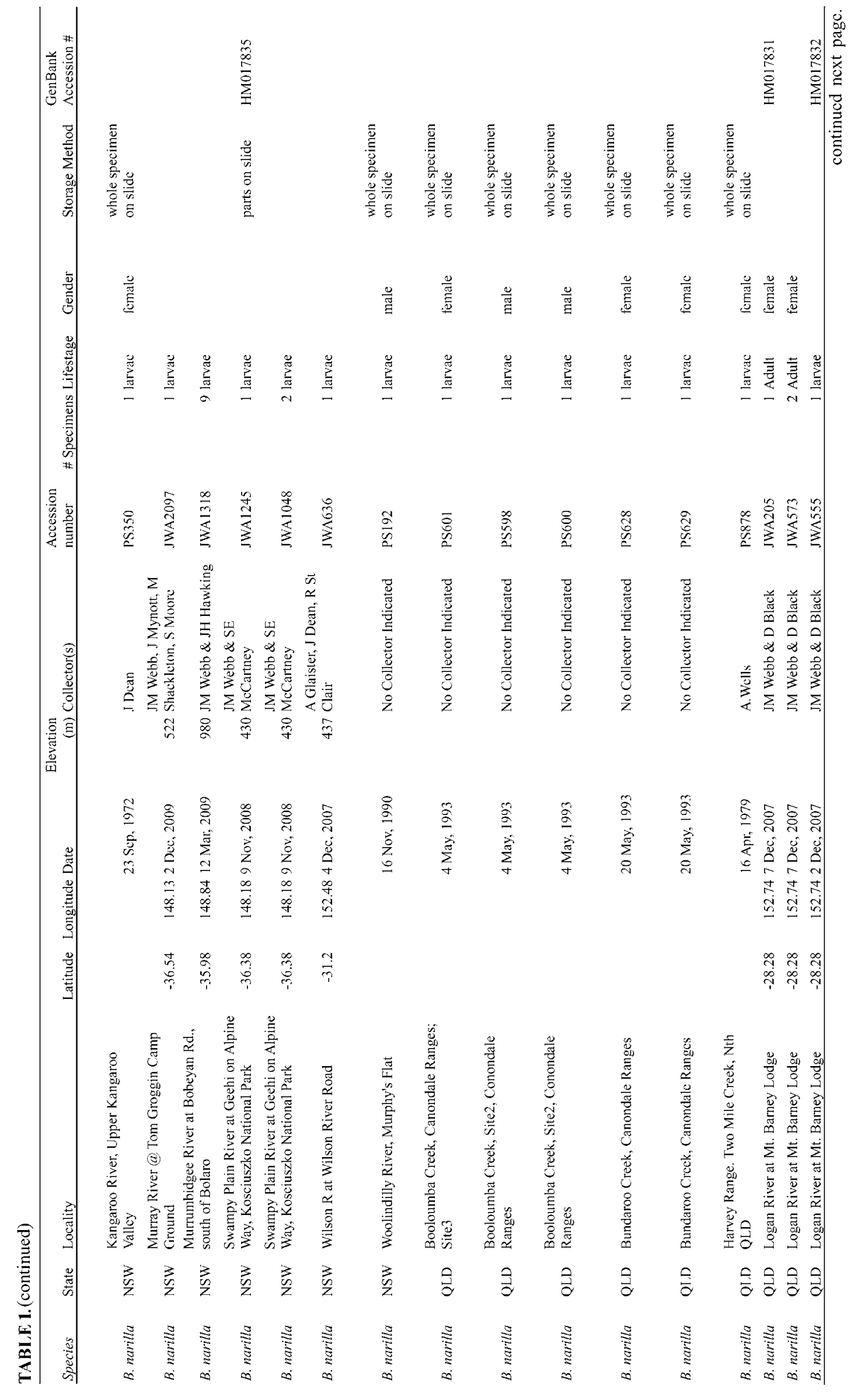




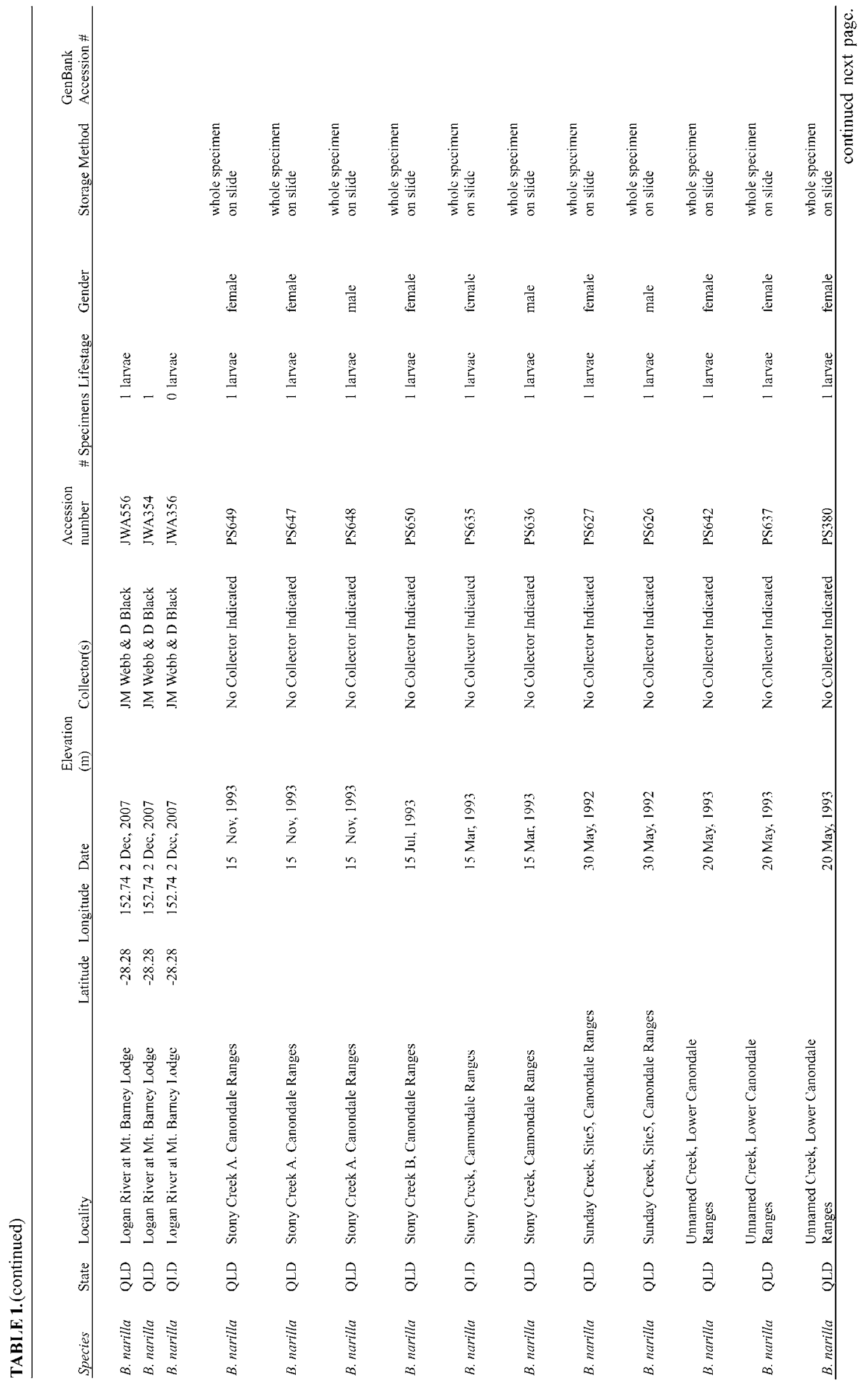




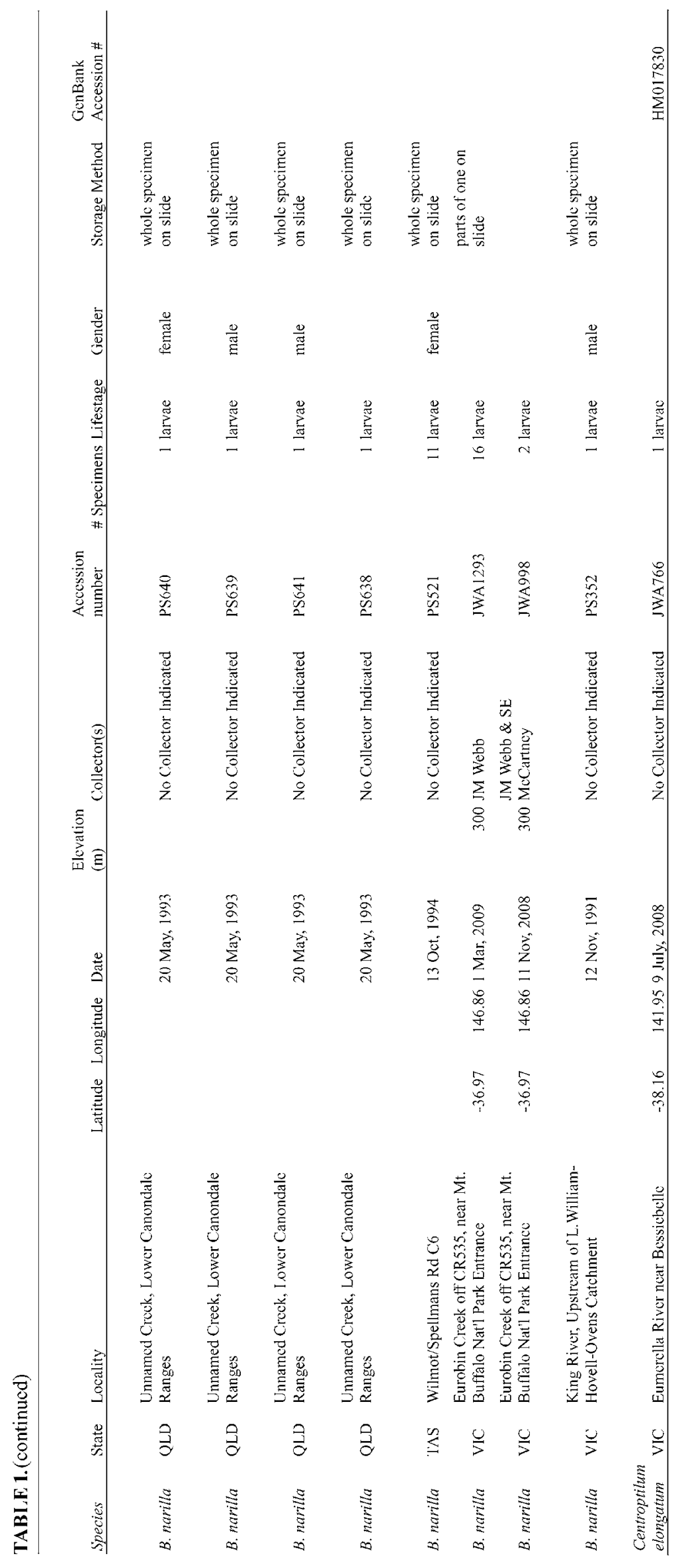


swapping. Bootstrap support was calculated with 1000 replicates with 10 random sequence addition replicates and TBR. Trees were rooted using a sequence of Centroptilum elongatum Suter, 1986 (GenBank accession number HM017830 ). Unique, fixed bases for each species were identified manually.

In order to obtain a better view of variation within B. narilla, 33 haplotypes of a 397-bp fragment of COI of B. narilla were obtained from McLean et al. (2008) (GenBank accession numbers EU789591-EU789623). A NJ analysis using the 397-bp fragment of all specimens was produced and p-distances recalculated.

\section{Results}

There were no double peaks in the chromatograms and no frame shifts, indels, or stop codons were observed in any of the 12 sequences. Two haplotypes were observed in northern specimens, and four in southern specimens. Specimens of Bungona from northern Queensland had two haplotypes and were strongly supported as being distinct from southern populations in both NJ (Fig. 21) and MP (not shown) reconstructions. Between group divergence (p-distance) was 18.8-19.4\% (mean 19.0\% $\mathrm{n}=12$ ) and within group divergence ranged from $0.0-0.2 \%$ (mean $0.04 \% \mathrm{n}=8$ ) in northern populations, and from $0.31-1.7 \%$ (mean $1.0 \% \mathrm{n}=4$ ) in southern populations. Of the 657 base positions, there were 129 variable sites. The northern populations had 115 fixed differences from the southern populations (Table 2).

All southern specimens clustered within the 33 haplotypes of a 397-bp fragment of COI from B. narilla identified by McLean et al. (2008) (Fig. 22). All of the haplotypes in our southern specimens differed from those of McLean et al. (2008), except JWA205 from southeast Queensland which was equivalent to "haplotype 6"/EU789594. Using the 357-bp fragment, mean within group divergence was $1.3 \%$ in $B$. narilla and $0.1 \%$ in northern populations, and mean intergroup divergence was $20.1 \%$.

TABLE 2. List of unique fixed character states in a 657-bp fragment of COI for B. illiesi and B. narilla.

\begin{tabular}{ll}
\hline Species & Position:Base \\
\hline B. illiesi & 6:A;30:T;33:A;39:G;45:A;51:G;54:A;55:C;57:T;58:G;60:T;66:G;75:A;78:A;93:C;108:G;114:T;121:G;1 \\
& 29:T;135:T;144:C;153:T;156:C;168:T;180:A;183:T;189:A;192:C;205:T;207:G;211:T;213:A;216:T;222: \\
& G;228:G;231:T;234:T;240:A;243:A;249:C;258:C;261:A;265:C;276:T;283:C;285:T;286:C;289:A;291:T; \\
& 294:A;300:T;303:A;306:A;309:T;310:A;315:G;318:T;324:A;327:A;333:T;336:C;342:A;345:G;346:C;34 \\
& 8:T;351:G;363:T;369:A;378:G;381:T;405:C;411:T;414:T;417:T;420:A;438:C;450:T;453:A;456:A;459:T \\
& ;462:T;465:A;471:A;474:T;480:G;481:A;492:A;495:A;501:A;507:G;513:A;516:T;519:G;531:C;534:A;5 \\
& 41:T;543:G;552:A;555:T;558:G;561:G;564:A;570:T;577:C;579:T;582:T;588:T;595:T;597:A;603:C;606: \\
& T;615:T;618:T;621:C;627:C \\
& 6:G;30:A;33:T;39:T;45:T;51:A;54:T;55:T;57:G;58:T;60:A;66:T;75:G;78:T;93:T;108:A;114:C;121:A;12 \\
& 9:A;135:C;144:T;153:C;156:T;168:A;180:T;183:G;189:T;192:T;205:C;207:T;211:C;213:T;216:G;222:A \\
& ;228:A;231:C;234:C;240:T;243:G;249:T;258:T;261:G;265:T;276:A;283:T;285:A;286:T;289:G;291:A;29 \\
& 4:T;300:A;303:G;306:T;309:C;310:G;315:A;318:G;324:G;327:G;333:A;336:G;342:T;345:T;346:T;348: \\
& A;351:T;363:C;378:T;381:A;405:T;411:A;414:G;417:G;420:T;438:A;450:C;453:T;456:T;459:C;462:C; \\
& 465:G;471:C;474:A;480:A;481:T;492:T;495:T;501:G;507:C;513:G;516:A;519:T;531:A;534:G;51:C;54 \\
& 3:A;552:T;555:G;558:A;564:T;570:C;577:T;579:G;582:A;588:C;595:C;603:T;606:A;615:C;618:G;621: \\
& T;627:A
\end{tabular}

\section{Taxonomy}

\section{Bungona illiesi (Lugo-Ortiz \& McCafferty, 1998)}

Cloeodes illiesi; Lugo-Ortiz \& McCafferty 1998: 124 (orig.)

Bungona narilla; Suter and Pearson 2001: 249 (=C. illiesi) 

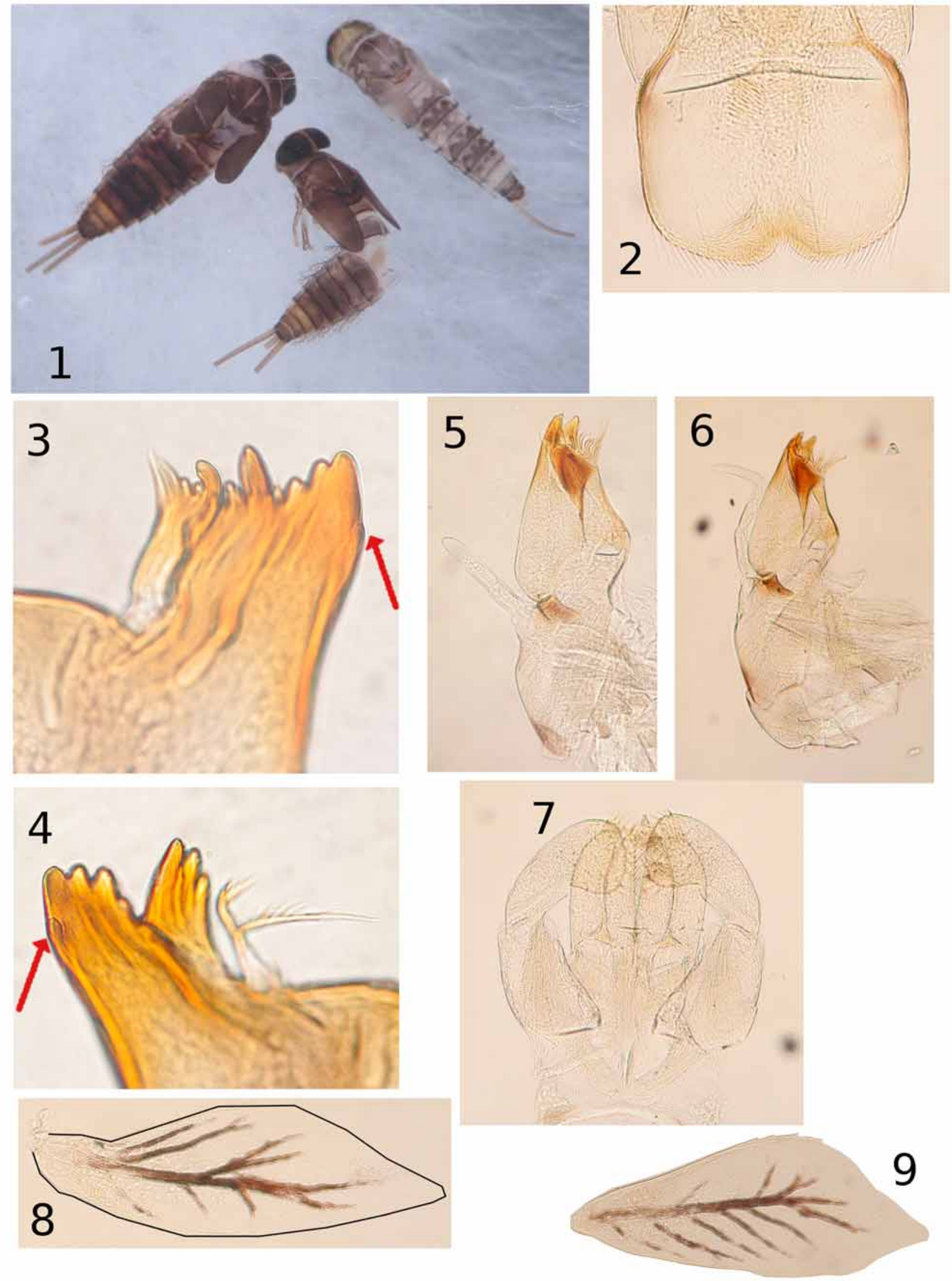

FIGURES 1-9. Bungona illiesi. 1. dorsal habitus of three larvae, 2. labrum, dorsal view, 3. left mandible with small lateral tooth indicated, 4. right mandible with small lateral tooth indicated, 5. maxilla showing aberrant minute palp, 6 . maxilla from same specimen as Fig. 5 showing normally developed palp, 7. labium, 8. gill 1 with margins outlined, 9. gill 7. 


\section{Description}

Larva: Body length: 3.1-3.8 mm, female larvae slightly larger than males. Caudal filaments $\sim 0.5 \mathrm{x}$ body length.

Head mostly brown; scape and pedicel subequal in length. Labrum (Fig. 2) with length subequal to width; marginal fringe of setae highly branched apically; submarginal fringe with 1+4-5 long simple setae. Right mandible (Fig. 4) with incisors separated apically, outer incisor with 1+3-4 teeth, inner incisor with 3 teeth; prostheca forked; mola with a single fimbriate seta on medial margin; area between prostheca and mola with or without small spines. Left mandible (Fig. 3) with incisors fused to apex, outer incisor with 1+4 teeth, inner incisor with 3-4 teeth; prostheca unforked and robust with apical denticles; area between prostheca and mola with or without small spines. Hypopharynx with superlingua densely covered with fine setae apically, with stout setae laterally becoming stouter basally; lingua with small median lobe covered with fine setae (LugoOrtiz and McCafferty 1998, Fig. 11). Maxillae with two segmented palps reaching the apex of the galealacinia, first segment approximately 0.3 times length of galealacinia, second segment sometimes appearing three segmented or may be minute. Galealacinia with 1+3-4 setae basally and single seta near apex. Labium with palp segment 3 slightly expanded medially.

Thorax mostly brown, median line pale. Femora (Fig. 10) with 5-7 long, blunt robust setae and subapical pair of long, blunt robust setae on dorsal margin; apex slightly sinuate and with short fine setae; anterior surface with scale bases, ventrally with 5-9 sharply pointed robust setae basally; posterior surface with scattered fine and short pointed robust setae near ventral margin and many circular chloride cells. Tibiae (Fig. 10) with dense arc of very long, fine setae on outer surface, continuing over outer margin near base onto posterior surface; inner margin with 7-9 short, pointed robust setae. Tarsi (Fig. 10) with 5-9 short, robust setae all of approximately the same length on inner margin; outer margin without robust setae; outer surface with dense row of very long, fine setae.

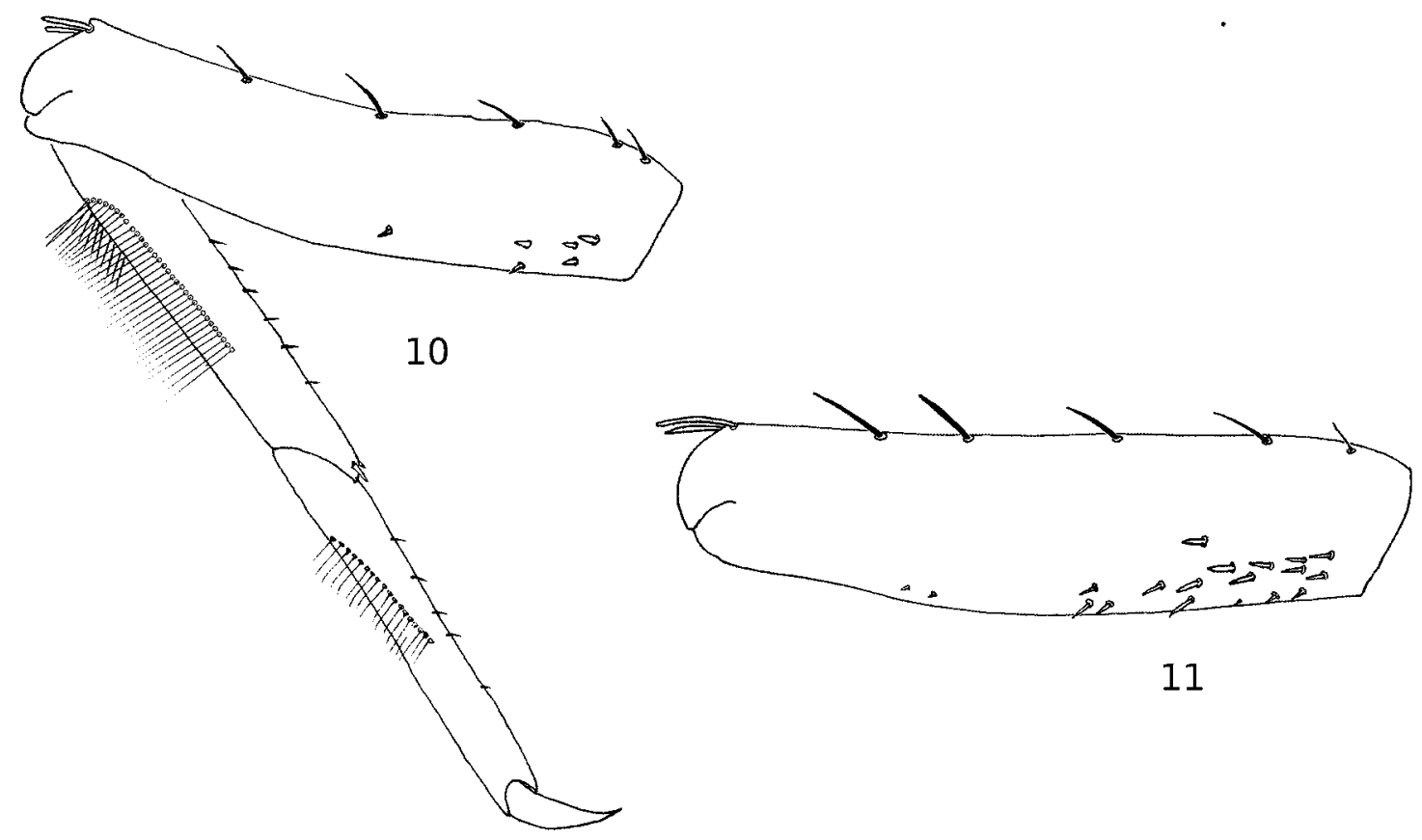

FIGURES 10-11. 10. foreleg of Bungona illiesi, 11. forefemur of Bungona narilla.

Abdomen with gills present on segments 1-7; gill 1 narrow-elongate (Fig. 8), margin smooth; gills 2-7 ovate to narrow elongate, margins serrate (Fig. 9). Colour pattern variable (Fig. 1), usually mostly brown with segments 4 and 8 paler. Terga 2-10 with sharply pointed spines on middle $2 / 3$ of posterior margins; surface with many triangular scales, scale bases, and sparse fine setae; tergum 1 without spines on posterior margin. Sterna pale; posterior margins of sterna 5-9 with long sharp spines approximately 1.5 times longer than wide 
in middle 1/3. Paraprocts with many scale bases and fine setae, margins with 11-13 sharp spines. Caudal filaments unbanded, cerci subequal in length to terminal filament.

Male Adult (Fig. 12): Body length: 4.2-4.7 mm. Forewing 3.5-4.0 mm. Caudal filaments broken and missing.
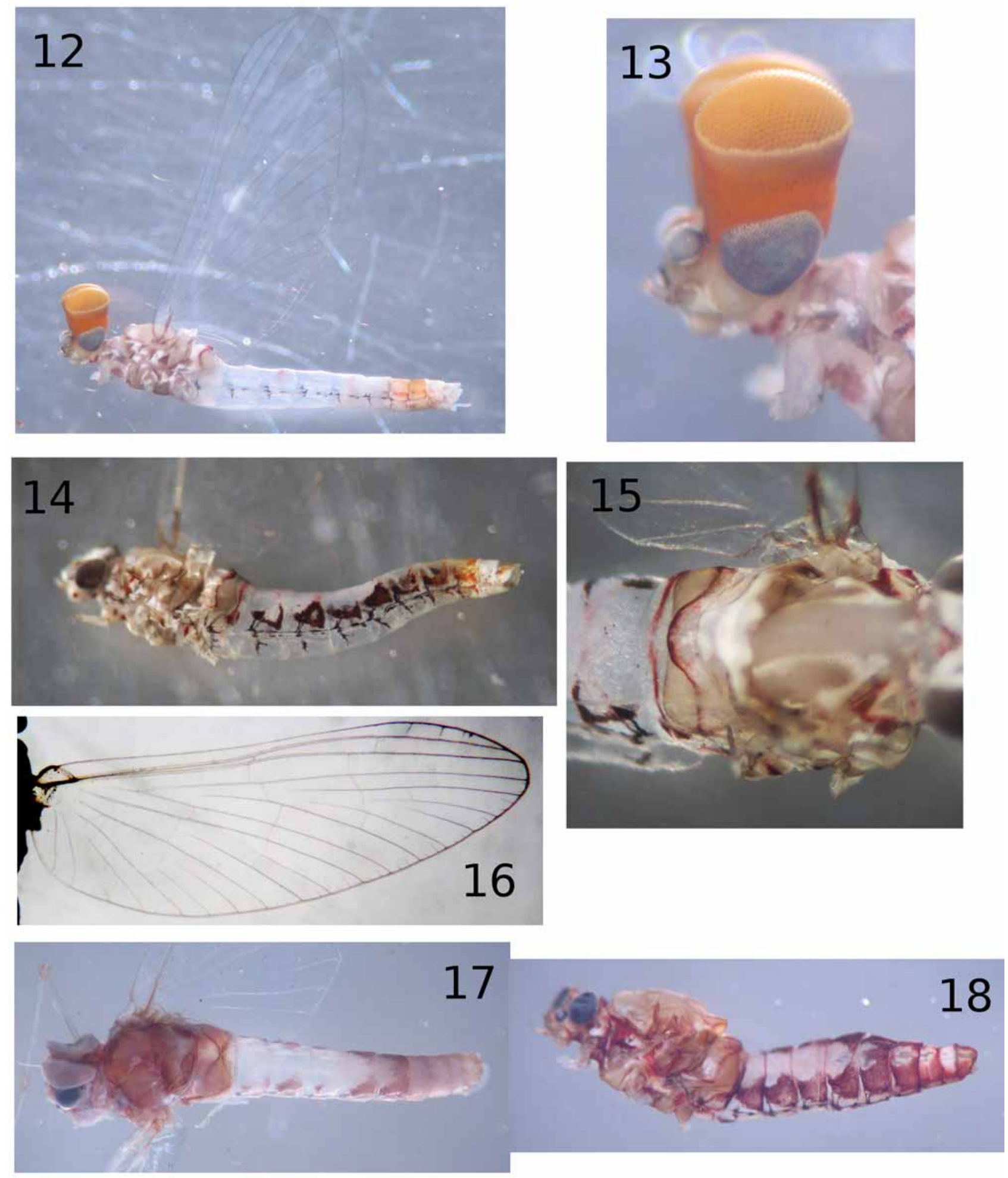

FIGURES 12-18. Bungona illiesi. 12. lateral view of male imago, 13. lateral view of head of male imago, 14. lateral view of female imago, 15. metascutellum of female imago with broadly rounded posterior margin, 16. wing of male imago; Bungona narilla. 17. dorsal view of male imago, 18. lateral view of female imago. 


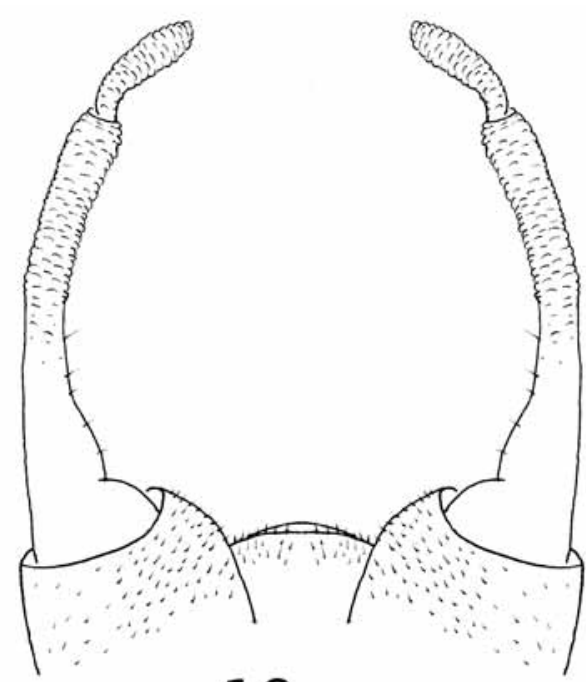

19

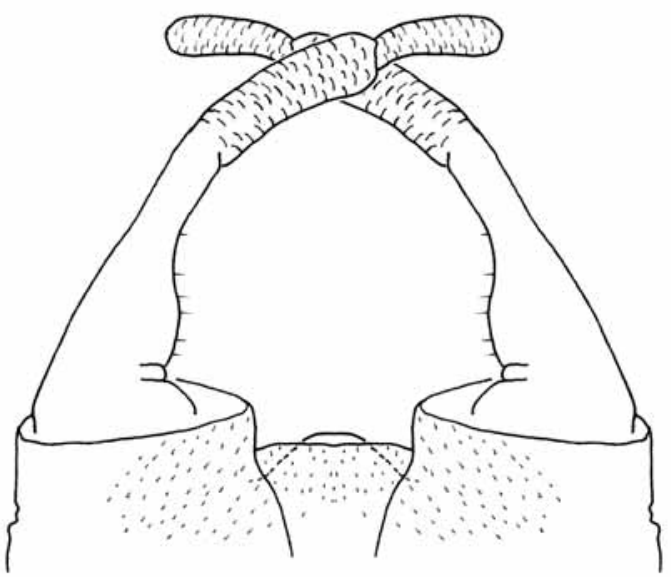

20

FIGURES 19-20. male genitalia. 19. Bungona illiesi, 20. Bungona narilla.

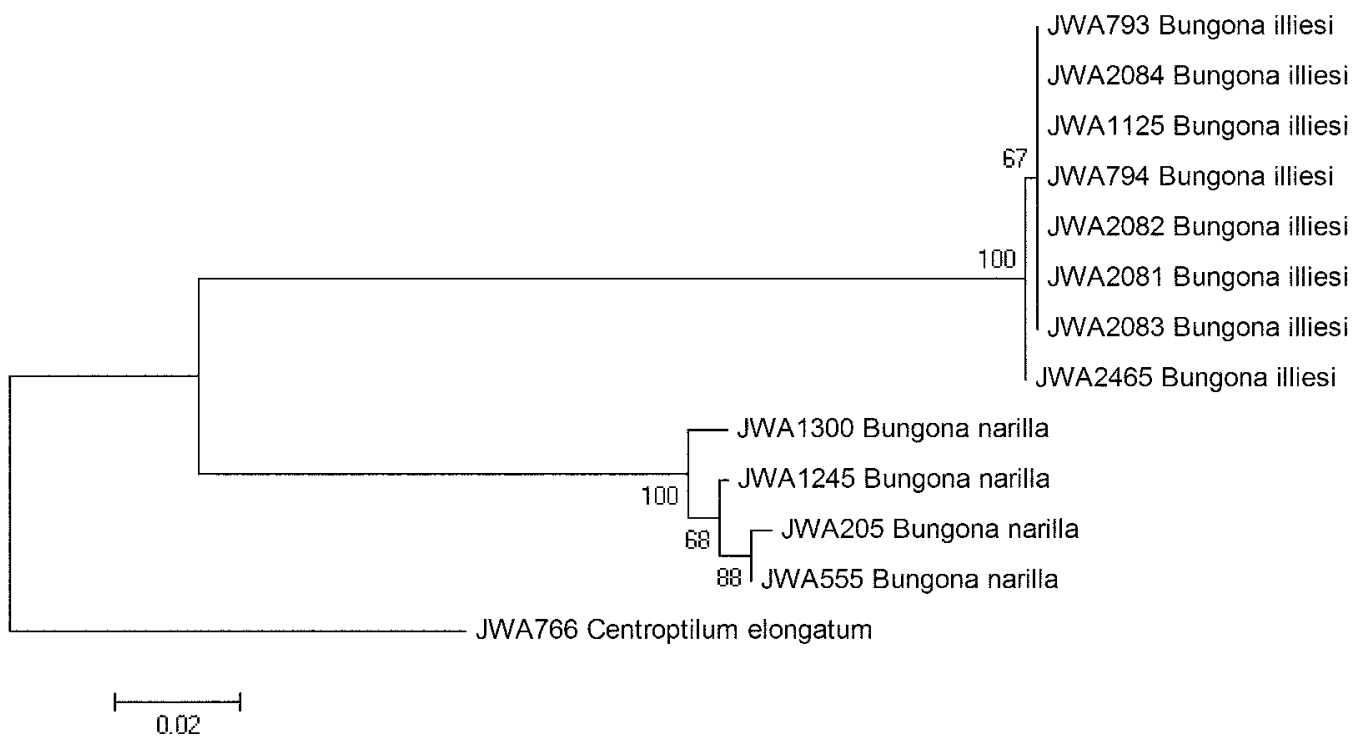

FIGURE 21. Neighbour-Joining tree of 657-bp fragment of COI of 12 specimens of Bungona rooted on Centroptilum elongatum, Kimura 2 parameter distance. Bootstrap values are indicated on branches.

Head yellow brown, turbinate eyes orange, tall and only slightly expanded distally (Fig. 13), contiguous basally and separated by distance subequal to width of lateral ocellus distally; compound eyes uniformly black. Pronotum and mesonotum cream to pale brown; mesoscutellum white. Forewings colourless; longitudinal veins slightly brown coloured; pterostigma whitish; intercalaries double distal of MP, single basally of MP; $\mathrm{MA}_{2}$ not attached to crossvein between MA and MP; area between $\mathrm{C}$ and Sc without crossveins basal of pterostigma. Hindwings absent. Posterior margin of metanotum concave. Metascutellum cream to pale brown, posterior margin red and broadly rounded, without dorsal projection. Thoracic sterna with sclerites pale brown to brown. Legs missing. Abdominal terga 1-6 white, terga 1, 3, 5 with faint transverse red stripe medially (Fig. 12); tergum 6 with distinct red marking laterally; terga 7-10 with faint pair of submedian red lines; terga 7 and 8 yellowish brown with red longitudinal streak laterally, terga 9 and 10 cream to yellowish brown. Forceps (Fig. 19) white, 3 segmented; separation between segments 1 and 2 indistinct, third segment approximately 3 times longer than wide, segments 2 and 3 covered with scale-like scalloping, segment 1 with small fine setae medially; forceps base and area between bases densely covered 
with small sharply pointed stout setae; small somewhat truncate projection dorsally between bases of forceps.

Female Adult (Fig. 14): Body length: 3.8-4.6 mm. Forewing 3.8-4.7 mm. Caudal filaments broken and missing.

Forewings (Fig. 16) similar to those of male except $\mathrm{MA}_{2}$ attached to crossvein between MA and MP Posterior margin of metanotum only slightly concave (Fig. 15); metascutellum cream with posterior margin red and broadly rounded, without dorsal projection. Abdominal terga (Fig. 14) white with reddish brown markings laterally and posterior margins with faint red transverse band.

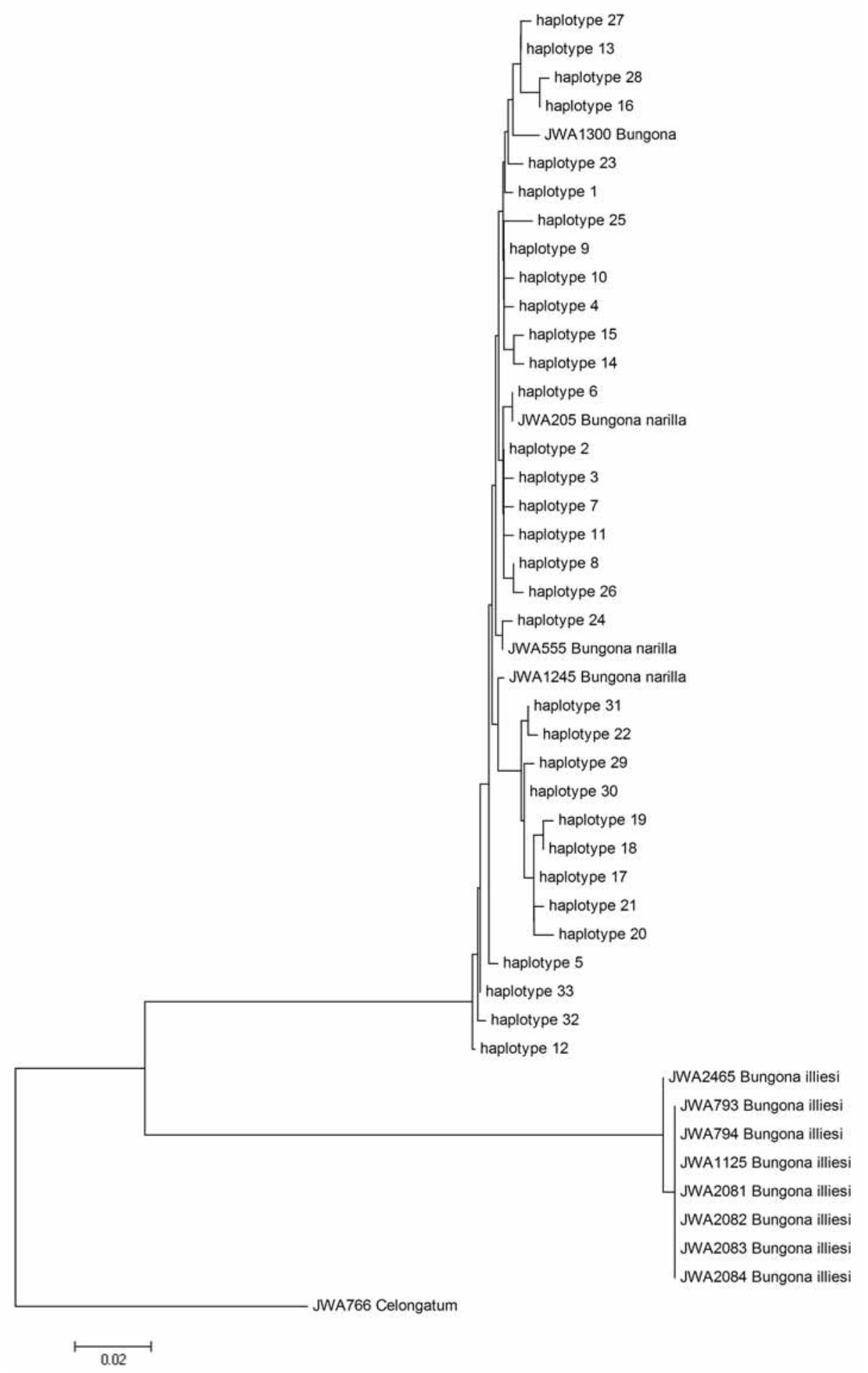

FIGURE 22. Neighbour-Joining tree of 397-bp fragment of COI of 12 specimens of Bungona together with 31 haplotypes (GenBank accession numbers EU789591-EU789623) of B. narilla from McLean et al (2008), rooted on Centroptilum elongatum, Kimura 2 parameter distance. 
Subimagos: Similar to adults in colouration.

Distribution: wet tropics and Atherton Tablelands of northern Queensland. The southernmost record is from near Tully, QLD. Bungona narilla occurs from Tasmania to southeast Queensland, but is expected to occur as far north as the Burdekin Gap north of Townsville, QLD, a recognised biogeographic division for aquatic insects (i.e. Christidis and Dean 2008, Watson and Theischinger 1984).

Diagnosis: Larvae of $B$. illiesi can be differentiated from those of $B$. narilla by a northern Queensland distribution, the presence of a small tooth on the outer margin of the set of incisors (Figs 3,4), and shorter and less dense robust setae at the base of the ventral margin of the fore femur (Fig.10). Previously identified diagnostic characteristics such as the absence of small spines on the mandibles between the mola and incisors, minute maxillary palps, the medial expansion of the labial palps, and narrow-elongate gills (Lugo-Ortiz and McCafferty 1998: Figs 12-15,17) were all found to be variable within populations of both B. illiesi and $B$. narilla.

Males and females of $B$. illiesi are easily differentiated from those of $B$. narilla by less extensive red patches on the abdomen (Figs. 12,14) and a northern Queensland distribution. Additionally, in the males of $B$. illiesi, the forceps are slightly more slender, the setae covering the bases of the forceps are longer, and $\mathrm{MA}_{2}$ is not attached to the crossvein between MA and MP.

Material examined: see Table 1.

\section{Discussion}

Bungona shares many similarities with Cloeodes, including small transverse rows of setae on the abdominal sterna, a subproximal arc of fine setae on the tibae, edentate claws, two segmented maxillary palps with the second segment having a constriction, right mandible with apically separated incisors and a forked prostheca, left mandible with apically fused incisors and a robust prostheca with apical denticles. Some of the diagnostic characters for Cloeodes are known to vary, however. For example, C. pseudogladius Gattolliat, 2001 has highly modified, completely fused incisors (Gattolliat 2001) and C. bicoloratus Gattolliat, 2001 and C. freitagae Gattolliat, 2001 have an unforked right prostheca (Gattolliat 2001). Larvae of Bungona differ from Cloeodes by having the ventral sternal setae only on segments 4-6, rather than 2-6. The incisors of the left mandibles of Bungona are nearly always fused to the apex or near the apex, but at least one specimen of $B$. narilla has elongate teeth on the outer incisor that make it appear that the two incisors are apically separate. Many specimens of $B$. narilla appear to have three segmented maxillary palps, but it is unclear if this is due to the second segment folding or collapsing during slide mounting or if they are actually three segmented.

Adults of Bungona differ from Cloeodes by having the third segment of the forceps elongate rather than rounded, although $C$. inzingae (Crass, 1947) from South Africa also has an elongate third segment (Waltz and McCafferty 1994), and by the absence of a dorsal projection on the metascutellum. Suter and Pearson (2001) indicated Bungona differed from the known species of Cloeodes by having $\mathrm{MA}_{2}$ attached to the crossvein between MA and MP, but in at least $B$. illiesi this varies between males and females. The recently described $C$. barituensis Nieto \& Richard, 2008 from Argentina shows the same sexual dimorphism in $\mathrm{MA}_{2}$ (Nieto and Richard 2008: Figs. 2,4).

A global revision is needed to clarify the relationships of Cloeodes, Bungona, and related genera as it is possible that Bungona is a specialised lineage within Cloeodes. Preliminary analyses of fragments of $18 \mathrm{~S}$ and 16S rDNA (Webb unpublished) from numerous Baetidae genera indicate Bungona and Afrotropical Cloeodes are relatively distantly related, despite their morphological similarity. Further analyses including Neotropical and Oriental Cloeodes and related genera, sequences of protein coding genes, and morphology are required, however, before the relationships among these groups can be inferred. Until these analyses are completed, we believe Bungona should be continued to be treated as a valid genus separate from Cloeodes. 


\section{Acknowledgements}

Special thanks are given to John Hawking for assistance in field work. Steve Moore and Darryl Rowe assisted in completing laboratory work. This research was undertaken as part of the Commonwealth Environmental Research Facilities sponsored Taxonomy Research \& Information Network.

\section{References}

Christidis, F. \& Dean, J. (2008) Phylogeny and distribution of the mayfly genus Austrophlebioides Campbell \& Suter (Ephemeroptera: Leptophlebiidae). Invertebrate Systematics 22, 29-36.

Crass RS. (1947) The May-flies (Ephemeroptera) of Natal and the Eastern Cape. Annals of the Natal Museum, 11, 37 110.

Folmer, O., Black, M., Hoeh, W., Lutz, R. \& Vrijenhoek, R. (1994) DNA primers for amplification of mitochondrial cytochrome c oxidase subunit I from diverse metazoan invertebrates . Molecular Marine Biology and Biotechnology 3, 294-299.

Harker, JE. (1957) Some new Australian Ephemeroptera. Part II. Proceedings of the Royal Entomological Society of London (B), 26, 69-78.

Gattolliat J-L. (2001) The genus Cloeodes (Ephemeroptera: Baetidae) in Madagascar. Revue Suisse de Zoologie, 108, $387-402$.

Lugo-Ortiz, C.R. \& McCafferty, W.P. (1998) First report and new species of the genus Cloeodes (Ephemeroptera: Baetidae) from Australia. Entomological News, 109, 122-128.

McLean, A.J, Schmidt, D.J \& Hughes, J.M. (2008) Do lowland habitats represent barriers to dispersal for a rainforest mayfly, Bungona narilla, in south-east Queensland? Marine and Freshwater Research, 59, 761-771.

Nieto, C. \& Richard, B. (2008) The genus Cloeodes (Ephemeroptera: Baetidae) in Argentina with new generic synonymy and new species. Zootaxa, 1727, 1-21.

Suter, P.J. (1986) The Ephemeroptera (mayflies) of South Australia. Records of the South Australian Museum, 19, 339397.

Suter, P.J. \& Pearson, M.J. (2001) Redescription of Bungona Harker with new synonyms in the Australian Baetidae (Insecta Ephemeroptera). Memoirs of Museum Victoria, 58, 247-254.

Swofford, D.L. (2002) PAUP* 4.0b10: Phylogenetic Analysis Using Parsimony (and other methods). (Sinauer Associates: Sunderland, MA, USA).

Tamura, K., Dudley, J., Nei, M. \& Kumar, S. (2007) MEGA4: Molecular Evolutionary Genetics Analysis (MEGA) software version 4.0. Molecular Biology and Evolution, 24, 1596-1599.

Waltz, R.D. \& McCafferty, W.P. (1994) Cloeodes (Ephemeroptera: Baetidae) in Africa. Aquatic Insects, 16, 165-169.

Watson, J.A.L. \& Theischinger, G. (1984) Regions of taxonomic disjunction in Australian Odonata and other freshwater insects. Odonatologica 13, 147-157. 